\title{
La mediación pedagógica como una estrategia para la atención educativa de las personas con enfermedad mental en el Programa de Educación Abierta, en el nivel de alfabetización que se imparte en el Hospital Nacional Psiquiátrico
}

\section{Pedagogical Mediation as an Educational Strategy for Students with Mental Illness in the Open Education Program of the National Psychiatric Hospital (Costa Rica)}

\author{
Karen Hidalgo Montoya ${ }^{7}$ \\ Escuela Fernando Centeno Güell, Departamento de Retardo Mental \\ Ministerio de Educación Pública \\ San José, Costa Rica \\ karenveli@yahoo.com \\ Virginia Navarro Solano ${ }^{2}$ \\ Servicio de Educación Especial , Colegio Técnico Profesional de Pacayas (CTPP) \\ Ministerio de Educación Pública \\ San José, Costa Rica \\ andrenavarro155@hotmail.com \\ María Roxana Rodríguez Araya ${ }^{3}$ \\ División de Educación Básica, Centro de Investigación y Docencia en Educación \\ Universidad Nacional \\ Heredia, Costa Rica \\ rodri156@gmail.com
}

Recibido 24 de julio de 2012 • Corregido 29 de enero de 2013 • Aceptado 13 de marzo de 2013

1 Licenciada en Educación Especial con énfasis en Integración de la Universidad Nacional. Bachiller en Educación Especial con énfasis en Integración de la Universidad Nacional. Ha ejercido cinco años en el Hospital Nacional Psiquiátrico en Pavas, para el servicio de Terapia Ocupacional de dicha institución. Se ha desempeñado por dos años en la escuela Fernando Centeno Güell en el Departamento de Retardo Mental del Ministerio de Educación Pública de Costa Rica, en la modalidad del Tercer ciclo con adolescentes. Actualmente labora en el servicio de estimulación temprana con bebés de 0 a 3 años en la misma institución.

2 Licenciada en Educación Especial con énfasis en Integración de la Universidad Nacional de Costa Rica. Bachiller en Educación Especial con énfasis en Integración de la Universidad Nacional de Costa Rica. Diplomado en Educación Básica en I y II ciclo de la Universidad Nacional de Costa Rica. Ha trabado durante tres años como docente de III ciclo en el Servicio de Educación Especial del Colegio Técnico Profesional de Pacayas (CTPP) del Ministerio de Educación Pública de Costa Rica (MEP), lugar donde labora actualmente.

3 Maestría Académica de la Universidad de Minnesota, certificado en Desórdenes del Espectro Autista de la Universidad de Minnesota. Bachiller en Educación Preescolar de la Universidad Estatal a Distancia de Costa Rica. Laboró como investigadora asociada en HACER (Hispanic Advocacy and Community Empowerment through research) y centros educativos dentro y fuera del país. Actualmente labora como académica en la Universidad Nacional, en la División de Educación Básica y en el Proyecto UNA Educación de Calidad. 
URL: http://www.una.ac.cr/educare

CORREO: educare@una.cr

Resumen. La investigación se enfoca en la atención que brindan el personal docente a los usuarios y usuarias del Programa de Educación Abierta que se ofrece en el Hospital Nacional Psiquiátrico e identifica las barreras existentes para el aprendizaje del estudiantado en el programa. El objetivo principal de la investigación es promover, por medio de la mediación pedagógica, una atención educativa ajustada a las necesidades de la población que asiste al nivel de alfabetización. El enfoque del estudio es cualitativo. El diseño utilizado fue el de investigación acción. Para recolectar información se utilizaron entrevistas dirigidas a los docentes, observación y la aplicación de una mediación pedagógica con las docentes del programa. Entre los principales resultados se puede mencionar que la metodología utilizada durante las mediaciones produjo una mejora en el acceso estudiantil al currículo. Como recomendación se requieren aplicar adecuaciones curriculares significativas a los estudiantes y una metodología de aula ajustada a la diversidad de los aprendizajes. Así mismo, se recomienda el establecimiento de un equipo interdisciplinario que provea de ayuda a las docentes que trabajan con estudiantes con enfermedad mental.

Palabras claves. Enfermedad mental, educación, mediación pedagógica, Revista Electrónica Educare, Costa Rica.

Abstract. This study focuses on the work of teachers in the Open Education Program of the National Psychiatric Hospital (Costa Rica) and identifies the learning barriers of the student population. It aims at promoting educational delivery strategies adapted to the needs of the community attending the literacy level. This is a qualitative study with a participatory action research design. Information was collected through interviews to teachers, observation, and pedagogical mediation with the teachers of the program. The results indicate that the pedagogical mediation methodology contributed to improve the access of students to the curriculum. Among the conclusions, it is recommended to implement significant curriculum modifications focused on the student population and a classroom methodology adjusted to the different learning needs. Finally, it is recommended to have an interdisciplinary team to support the teaching staff working with students with mental illness.

Keywords. Mental illness, education, pedagogical mediation, Educare Electronic Journal, Costa Rica.

\section{Introducción}

La atención educativa de las personas con enfermedad mental es un tema que ha sido abordado recientemente en Costa Rica. Este cambio se debe a una tendencia internacional de pasar de un modelo netamente institucionalista hacia uno donde se vislumbre a la persona con enfermedad mental como sujeto de derecho. Se procura, así, dejar de lado estereotipos que se centran en los síntomas y la peligrosidad de estas personas.

En muchas ocasiones la enfermedad mental conlleva, de forma adicional, la aparición de la discapacidad. Esto, debido al posible deterioro cognitivo del enfermo mental, así como a las condiciones sociales que, en algunas ocasiones, limitan sus posibilidades de integración social y de acceso a actividades socio laborales.

Es, entonces, necesario hacer referencia al tema de enfermedad mental desde un enfoque más global, en el cual se concibe a la persona con enfermedad mental como quien, a raíz de su condición de salud, puede, de forma asociada, presentar una discapacidad que repercute 
en sus actividades cotidianas. En esta línea, el Manual de recursos de la OMS sobre salud mental, derechos humanos y legislación (Organización Mundial de la Salud [OMS], 2006) cita a los autores Bertolote y Sartorius (1996) quienes definen la discapacidad mental como la que:

(...) incluye a las personas con trastornos mentales. Las personas que se han recuperado de un trastorno mental pueden seguir teniendo discapacidades, y muchas personas que experimentan trastornos mentales también tendrán discapacidades debidas al trastorno. "Discapacidad" significa, en algunas circunstancias, un signo intrínseco de una enfermedad o síndrome específico (por ejemplo, el diagnóstico de algunos trastornos mentales requiere la presencia de limitaciones funcionales), y en otros casos es una consecuencia de esa enfermedad o síndrome. (p. 25)

Por lo anterior, la persona con enfermedad mental debe concebirse amparada por las políticas nacionales e internacionales relacionadas a discapacidad. La discapacidad puede verse como conceptos que evolucionan y que tienen una estrecha relación con el entorno social y cultural. Por ende, la Convención de los Derechos Humanos de las Personas con Discapacidad, que se convirtió en la Ley 8661, establece que "(...) la discapacidad es un concepto que evoluciona y que resulta de la interacción entre las personas con deficiencias y las barreras debidas a la actitud y al entorno que evitan su participación plena y efectiva en la sociedad, en igualdad de condiciones con las demás" (Asamblea Legislativa de la República de Costa Rica, 2008, p. 1).

Adicionalmente, la Ley 7600, en el Título II Capítulo I: Acceso a la Educación, en los artículos del 14 al 17 se refiere al derecho a la educación de la persona con discapacidad y su atención de acuerdo con las necesidades educativas. Se menciona, además, en el artículo 16, que "Las personas con discapacidad participarán en los servicios educativos que favorezcan mejor su condición y desarrollo, con los servicios de apoyo requeridos; no podrán ser excluidas de ninguna actividad" (Asamblea Legislativa de la República de Costa Rica, 1996, p. 12).

Considerando las políticas y marcos jurídicos internacionales y nacionales sobre la protección y los derechos que amparan a las personas con discapacidad y, por ende, a las personas con enfermedad mental que, asociadas a su condición de enfermedad, presentan discapacidad, han surgido iniciativas en el Hospital Nacional Psiquiátrico que pretenden el cambio paradigmático de atención meramente institucionalizada, a una que propicie la reinserción social y laboral de los usuarios del servicio. El Hospital Nacional Psiquiátrico ha elaborado programas de intervención integral con base en un plan de rehabilitación institucional orientado a brindar una mejor calidad de vida. Uno de los servicios que se les brinda a los usuarios es el programa de Educación Abierta. Esta modalidad educativa se ha convertido en una oportunidad de accesar a la educación, la cual podría convertirse en una herramienta para su proceso de vida independiente e inserción social. 
URL: http://www.una.ac.cr/educare

CORREO: educare@una.cr

El Ministerio de Educación Pública, a través del programa de Educación Abierta ha hecho llegar la educación a los usuarios del Hospital Nacional Psiquiátrico, con un equipo de trabajo constituido por docentes de I y II ciclos que imparten las cuatro materias básicas: Español, Ciencias, Estudios Sociales y Matemática, además profesoras de secundaria en materias como Inglés y Cívica.

A nivel nacional, el programa de Educación Abierta es dirigido a la población adulta que, por distintas razones, se encuentra excluida o en riesgo exclusión del sistema educativo: una de las intenciones del programa es que los adultos puedan terminar su formación básica y continúen con estudios posteriores.

Al concebirse la educación como un derecho fundamental, es importante analizar qué ocurre con el desarrollo del Programa de Educación Abierta en el hospital y el acceso que tienen los usuarios al currículo a través de la educación vigente. Desde la perspectiva de la educación especial, es esencial promover espacios de reflexión interdisciplinaria entre docentes de I y II ciclos y docentes de educación especial, para enriquecer las estrategias didácticas que las docentes desarrollan en el servicio. La educación especial es un área educativa que, en el ámbito de la atención a la diversidad, cumple un papel relevante en cuanto a adaptaciones y mejoras al currículo, según las características de la población (Sánchez, 1997).

\section{Un breve marco referencial}

\section{Conceptualización de la salud mental}

La salud ha sido reconocida como un derecho de la persona y esto ha permitido que, a través de los años, se desarrollen políticas internacionales así como nacionales que promueven el acceso a un sistema de salud que respondan a las necesidades de la población.

En la década de los noventa, la Organización Panamericana de Salud recomendó a los países darles prioridad a las temáticas sobre salud mental. El Consejo Directivo de la Organización Panamericana de Salud/Organización Mundial de la Salud (OPS/OMS) aprobó la Resolución CD49.17, en el año 2009. En esta insta a los estados miembros "a) a que incluyan la salud mental como una prioridad dentro de las políticas nacionales de salud, mediante la ejecución de planes de salud mental que estén acordes con las diversas problemáticas y prioridades de los países (...)" (OPS y OMS, p. 1).

En esta misma resolución, la OPS exhorta a los países a continuar la lucha para "fortalecer los marcos legales con miras a proteger los derechos humanos de las personas con trastornos mentales para lograr la aplicación eficaz de las leyes" (p. 1).

Nuevamente, la OPS en el 2010, realiza una nueva resolución, la CD 50.R8, Ilamada La Salud y los Derechos Humanos, en esta pide, a los estados miembros, promover la salud de grupos en situaciones vulnerables tales como: personas con $\mathrm{VIH}$, poblaciones indígenas, personas con discapacidad y personas con enfermedad mental. Específicamente llama a que se: 
e) formulen y, de ser posible, adopten medidas de carácter legislativo, administrativo, educativo y de otra índole para difundir los instrumentos internacionales de derechos humanos que sean aplicables sobre la protección del derecho al goce del grado máximo de salud que se pueda lograr y otros derechos humanos relacionados entre el personal apropiado de los poderes legislativos, judiciales y otras autoridades gubernamentales.

f) promuevan la difusión de información entre las organizaciones de la sociedad civil y otros actores sociales, cuando corresponda, con respecto a los instrumentos internacionales de derechos humanos que sean aplicables relacionados con la salud, a fin de abordar la estigmatización, la discriminación y la exclusión de los grupos en situación de vulnerabilidad. (Organización Panamericana de la Salud, 2010, p. 3)

En cada una de las resoluciones que abordan la temática de salud mental, se identifica la necesidad de que se consideren y se aborden factores contextuales con políticas tendientes a eliminar la exclusión y la estigmatización de la persona con enfermedad mental. Por lo anterior, en la actualidad en el hospital se trabaja con un modelo de atención basado en la dinámica de la comunidad, a través de servicios idóneos que atiendan la enfermedad mental de la persona desde en el propio entorno social donde se ha desarrollado y vive el sujeto. Actualmente el Programa de Educación Abierta responde a esta nueva visión de ofrecer a las personas con enfermedad mental una atención educativa mientras viven en un contexto comunitario.

\section{Programa de Educación Abierta en el Hospital Nacional Psiquiátrico}

El programa de Educación Abierta en Costa Rica surge de la Política Educativa Hacia el siglo XXI, del Ministerio de Educación Pública (1994), como respuesta a lo establecido en el marco de Acción de Dakar. Educación para todos (UNESCO, 2000).

En el año de 1994 se aprobó en Costa Rica La Política Educativa Hacia el Siglo XXI que, según Arroyo y Chávez (2001), establece "que el desarrollo de la nación se da por la transmisión de conocimientos, valores éticos y es el medio generador de capacidades y destrezas indispensables para la competitividad mundial" (p. 324), para lo cual se plantea una serie de cambios en donde todos los elementos prácticos (programas de estudio, normativa de evaluación, libros, el trabajo en el aula, capacitación docente) sirvan para orientar el desarrollo social, como la generación de equidad social.

Entre los retos de la Política Educativa Hacia el Siglo XXI se mencionan: destinar recursos económicos a los sectores más vulnerables de la sociedad, aumentar el nivel cognitivo por medio de planes y programas destinados a ello y eliminar desigualdades en el sistema educativo (étnico, económico, político entre varios). (Ministerio de Educación Pública [MEP], 1994, p. 5) 
URL: http://www.una.ac.cr/educare

CORREO: educare@una.cr

El programa de Educación Abierta en el Hospital Nacional Psiquiátrico responde a la iniciativa de atender personas con algún grado de vulnerabilidad social, en este caso, las personas con enfermedad mental. Aunque la política educativa Hacía el Siglo XXI se inició en el año 1994, el programa en el Hospital Psiquiátrico no se inicia sino hasta el año 2008. La iniciativa se originó en el hospital, ya que existía la necesidad de que los usuarios y usuarias se dedicaran a una ocupación como parte de un proceso de reinserción social. Sin embargo, se evidenció que para que estas personas enviaran un currículo a una empresa, necesitaban habilidades como la lectoescritura y matemática, entre otras. Es, entonces, cuando se reconoce que estos usuarios requieren un apoyo en el área educativa o necesitan iniciar el proceso de educación formal.

En esta línea, Sandoval-Chacón y Canales-Víquez (2009) comentan que:

Sin duda, la condición de analfabetismo de los (as) [sic] usuarios debe motivar procesos educativos formales de la mano a los procesos rehabilitativos intrahospitalarios, pues la ausencia de instrucción constituye una lamentable limitación para una reinserción exitosa en el plano sociolaboral (p. 131).

A raíz de esta iniciativa que surge en el hospital, se comenzó a establecer los contactos con el Ministerio de Educación Pública, a través de cartas, reuniones y solicitudes, con el propósito de mostrar la creciente necesidad de capacitar a los usuarios y usuarias, principalmente en la lectoescritura y operaciones matemáticas básicas.

El Ministerio accede a brindar las clases del programa de Educación Abierta en el Hospital Nacional Psiquiátrico, en especial porque existían facilidades de infraestructura y espacios disponibles. Dentro del programa de Educación Abierta que se ofrece en el Hospital Nacional Psiquiátrico se encuentran diferentes niveles académicos para los estudiantes. El Proyecto de Educación Abierta contempla las cuatro materias básicas y formación ciudadana e inglés. La aprobación de los estudios se realiza mediante la aprobación de la nota mínima de las pruebas nacionales de conclusión del I y II ciclos de la Educación General Básica.

Sin embargo, existe evidencia de que el programa actual carece de una serie de condiciones que verdaderamente respondan a la realidad de los usuarios, tales como la carencia de adecuaciones curriculares, la obligatoriedad de realizar los exámenes anuales del MEP, la falta de capacitación docente para la atención de las necesidades educativas de los estudiantes a raíz de su condición mental, así como poco seguimiento de las labores que realizan las docentes. 


\section{Educación especial y su relación con la atención de personas con enfermedad mental}

Sin duda, la educación especial ha evolucionado a lo largo de los años en sus concepciones sobre discapacidad como respuesta a un cambio social que transita desde un modelo tradicional hasta un modelo social (Molina, 2003). La educación especial al servicio de la persona con enfermedad mental permite la potencialización, y el desarrollo de habilidades educativas y sociales del individuo, respetando la diversidad personal que lo caracteriza. En esta línea, Pinto (s. f.) menciona que "la Educación Especial se considera como diversificadora de la respuesta educativa según las necesidades individuales de cada alumno" (p. 5).

Desde la visión de la educación especial, el paradigma de atención debe seguir evolucionando hacia el reconocimiento de la diversidad educativa, la cual se fundamenta en el hecho de que todos los estudiantes son diferentes y deben ser reconocidos en su singularidad y valoradas sus posibilidades de participar y de aprender de acuerdo con sus capacidades.

En esta línea Williamson, (2007) manifiesta que:

Crece la comprensión respecto de la diversidad en la educación. Hoy es una exigencia de cualquier política, programa o proyecto el referirse a esta dimensión de la especie humana, su vida social y su individualidad. Trabajar la diversidad en el aula es al mismo tiempo un principio pedagógico y un requerimiento para alcanzar mejores y mayores aprendizajes (...). (p. 58)

La diversidad es una característica intrínseca de los grupos humanos, ya que cada persona tiene una forma diferente de pensar, de sentir y de actuar, independientemente de que existan unos patrones cognitivos, afectivos y conductuales con ciertas diferencias. Dicha variabilidad se vincula con capacidades, necesidades variadas, intereses, condiciones socioculturales, entre otras.

La diversidad es algo positivo, es el soporte para el aprendizaje y el desarrollo. Según Cabada (2004),"la diversidad enfatiza el papel de la [educación] como agente de transformación social, (...) enfocar la diversidad como un recurso y una oportunidad para el aprendizaje (...)". (La diversidad, párr. 2). El reconocimiento de la diversidad del educando con una enfermedad mental, así como su derecho a recibir una educación inclusiva, no puede reducirse a una cuestión de currículo, organizativa o metodológica; la inclusión es más que todo eso, es una manera distinta de entender la educación y, si se quiere, la vida misma y la sociedad: se trata más bien de una filosofía, de valores (Lamas y Murrugarra, s. f.).

Según Giné (s. f.), siguiendo a Ainscow (2000), Faro y Vlageliu (2000) y Porter (2000), los factores considerados para construir una educación inclusiva son: 
URL: http://www.una.ac.cr/educare

CORREO: educare@una.cr

- Partir de la experiencia y conocimientos propios; de sus éxitos, que a menudo son muchos, y también de sus dificultades (...).

- Hacer del trabajo colaborativo el instrumento metodológico habitual para generar conocimiento que sirva de forma eficaz para responder a las diferentes necesidades del alumnado.

- Entender las dificultades (la heterogeneidad presente en las aulas) como oportunidades para diversificar la respuesta educativa (...) que supone el trabajo cooperativo de los alumnos y la colaboración entre iguales.

- Dotarse de una estructura de centro flexible, capaz de adaptarse a las características y necesidades del alumnado y del profesorado (...). (pp. 9-10)

Cuando se concibe la educación desde una perspectiva integral y se valora la individualidad del ser humano, se abre la puerta a la educación inclusiva, la cual toma en cuenta el contexto para influir en los procesos de aprendizaje específicos, con lo cual se incrementan las posibilidades de éxito del grupo estudiantil en sus situaciones de aprendizaje.

\section{El quehacer metodológico}

El tipo de investigación que se desarrolló fue de investigación acción, ya que “(...) se contemplan los problemas desde un punto de vista de quienes están implicados en ellos, (...) a través de un diálogo libre, (...) implica necesariamente (...) la auto reflexión sobre su situación" (Elliot, 2005, p. 26) Los participantes del estudio fue el personal docente del nivel de alfabetización del Programa de Educación Abierta del Hospital Nacional Psiquiátrico.

Para la recolección de la información se realizaron observaciones participativas y entrevistas a las docentes. Las entrevistas constaban de una serie de preguntas dirigidas a comprender las necesidades profesionales que tenían para atender a la población con enfermedad mental, así como las necesidades educativas más comúnmente encontradas en los estudiantes. Para responder a las necesidades encontradas, se realizó una serie de mediaciones pedagógicas. El objetivo de la mediación pedagógica consistió en que las docentes del Programa de Educación Abierta del Hospital Nacional Psiquiátrico tuvieran herramientas para atender y orientar las necesidades educativas de los estudiantes por medio de actividades que respondieran a las necesidades de la población. 
La mediación pedagógica se realizó en tres etapas:

\section{Construcción de planeamientos conjuntos}

Una de las debilidades identificadas en el proceso de observación fue el abordaje que dan las docentes a los contenidos por abarcar y las actividades de mediación realizadas. La mayoría de las ocasiones, los estudiantes no comprendían la materia porque se presentaba de una forma tradicional, con fotocopias, pero sin una acomodación metodológica a sus necesidades educativas. Por esto, las investigadoras se dieron a la tarea de planear, de forma conjunta con las docentes, y crearon oportunidades de utilizar actividades y materiales diferentes, más apropiados a las necesidades del estudiantado. Con cada maestra se realizaron ocho planeamientos y se promovieron actividades que permitieron compartir criterios y conocimientos de ambas partes.

Fue prioridad beneficiar las lecciones con creatividad y dinamismo, utilizando materiales concretos y de desecho. Se trató de dar atención y respuesta a los estudiantes tomando en cuenta la diversidad y los diferentes tipos de aprendizaje que hay en el aula.

\section{Implementación y mediación}

Luego del período donde se realizaron los planeamientos en conjunto, las investigadoras asistieron a las clases en las que las docentes aplicaron los planes realizados conjuntamente. La intención fue construir un proceso de gestión, en el cual los docentes se apoderan de las estrategias que conjuntamente habían creado. Existió un proceso de modelaje y de motivación hacia la construcción de aprendizajes significativos que respetaran las necesidades educativas del estudiantado.

\section{Autoevaluaciones del proceso}

Durante el período de aplicación de la serie de planes de trabajo en el aula, se realizó un proceso de autoevaluación de las docentes y de las investigadoras. Para esto se desarrollaron instrumentos en los cuales las docentes participantes y las investigadoras realizaron una autoevaluación del desempeño en la puesta en práctica de los planes, esto con el fin de auto reflexionar y crear una mejor práctica en lecciones futuras.

\section{Resultados}

Con el fin de organizar la información de una forma comprensible, se presentan las siguientes tablas, las cuales capturan los resultados obtenidos de las autoevaluaciones realizadas por las docentes e investigadoras. Para mayor claridad la información obtenida se categoriza. 
URL: http://www.una.ac.cr/educare

CORREO: educare@una.cr

Tabla 1

Elementos positivos que se identifican del proceso de mediación pedagógica, según docentes e investigadoras

\begin{tabular}{|c|c|c|}
\hline Categorías & Docentes & Investigadoras \\
\hline $\begin{array}{l}\text { Ritmos y estilos de } \\
\text { aprendizaje }\end{array}$ & $\begin{array}{l}\text { Se utilizan estrategias nuevas, hubo más } \\
\text { participación por parte de los estudiantes } \\
\text { y las estudiantes. } \\
\text { Las actividades van acordes con sus } \\
\text { necesidades educativas, por lo cual ellos y } \\
\text { ellas lo han aprovechado bastante. } \\
\text { Los periodos de atención y concentración } \\
\text { fueron más largos en los estudiantes. }\end{array}$ & \\
\hline Nivel cognitivo & $\begin{array}{l}\text { Se logra cumplir con los contenidos } \\
\text { establecidos, lo que permitió a la } \\
\text { mayoría de los estudiantes y las } \\
\text { estudiantes comprender la materia. } \\
\text { Las técnicas de aplicación vivencial } \\
\text { son muy buenas, ya que se unieron } \\
\text { conocimientos y se trabajó en conjunto } \\
\text { con los estudiantes y las estudiantes. } \\
\text { Se refuerzan en gran manera las } \\
\text { actividades de la semana y sirven de } \\
\text { práctica para ellos y ellas. }\end{array}$ & $\begin{array}{l}\text { Las actividades mejoraron el proceso de } \\
\text { la lectoescritura en los estudiantes y las } \\
\text { estudiantes. } \\
\text { Adicionalmente los estudiantes y las } \\
\text { estudiantes lograron comprender mejor } \\
\text { el tema de las fracciones, por medio de } \\
\text { las actividades vivenciales. }\end{array}$ \\
\hline Intereses y motivación & $\begin{array}{l}\text { Los estudiantes y las estudiantes } \\
\text { se sintieron muy motivados con las } \\
\text { actividades realizadas. } \\
\text { A los estudiantes y las estudiantes } \\
\text { les gustaron las actividades que se } \\
\text { desarrollan, les agradó mucho y } \\
\text { aprendieron bastante. }\end{array}$ & $\begin{array}{l}\text { Se da mayor participación en los } \\
\text { estudiantes y las estudiantes. } \\
\text { Las docentes se motivaron a expresar } \\
\text { nuevas ideas, así como a reflexionar y a } \\
\text { analizar la intervención }\end{array}$ \\
\hline
\end{tabular}

Nota. Propiedad de Hidalgo K., Navarro V. y Rodríguez R. (2012)

Un elemento positivo es la utilización de estrategias nuevas a las comúnmente utilizadas en las clases, como el uso de material concreto en donde el estudiantado puede aprender haciendo y asociando el significado de las palabras con los objetos. Además de ello se usaron materiales atractivos tales como fotografías, objetos con texturas, material audio visual como videos, presentaciones en power point, etc. Estos materiales fueron de gran ayuda durante el desarrollo de las lecciones, ya que se utilizó en menor medida el material fotocopiado.

Al variar las técnicas de trabajo, se permitió repasar conceptos, relacionando los conocimientos anteriormente aprendidos con las actividades de clase. Así mismo, los estudiantes y las estudiantes demostraron mayores períodos de concentración, lo que generó motivación para realizar las actividades: Se mostraron atentos al trabajo en clase, realizaban preguntas y comentarios con respecto a los contenidos desarrollados. 
Tabla 2

Elementos que limitaron el proceso de mediación pedagógica, según docentes e investigadoras

\begin{tabular}{|c|c|c|}
\hline Categorías & Docentes & Investigadoras \\
\hline $\begin{array}{l}\text { Ritmos y estilos de } \\
\text { aprendizaje }\end{array}$ & $\begin{array}{l}\text { Las actividades duraban más tiempo } \\
\text { del planeado, sobre todo por el poco } \\
\text { desarrollo de la motora fina. } \\
\text { Existió la necesidad de adaptar los } \\
\text { materiales en las actividades para los } \\
\text { estudiantes y las estudiantes de acuerdo } \\
\text { con los niveles de funcionamiento. }\end{array}$ & $\begin{array}{l}\text { Se debió dar mayor tiempo en las } \\
\text { actividades a los estudiantes y las } \\
\text { estudiantes, por lo que no se realizaron } \\
\text { los planeamientos completamente. } \\
\text { En este proceso había estudiantes } \\
\text { más avanzadosy avanzadas, por lo } \\
\text { que debían esperar a que el resto } \\
\text { comprendiera las actividades. }\end{array}$ \\
\hline Nivel cognitivo & $\begin{array}{l}\text { Algunos y algunas estudiantes no } \\
\text { recuerdan la información vista en clases } \\
\text { anteriores, por su memoria a corto } \\
\text { plazo. } \\
\text { Algunos y algunas estudiantes no } \\
\text { saben leer, lo que dificulta el proceso. } \\
\text { Se debe de explicar muchas veces las } \\
\text { cosas y de varias maneras. }\end{array}$ & $\begin{array}{l}\text { Se tiene que retomar el tema de la } \\
\text { lección anterior. } \\
\text { Los estudiantes y las estudiantes } \\
\text { olvidan en ocasiones la dinámica de las } \\
\text { actividades. } \\
\text { Los estudiantes y las estudiantes } \\
\text { requieren apoyo adicional para } \\
\text { comprender las actividades. }\end{array}$ \\
\hline Aspectos personales & $\begin{array}{l}\text { La situación personal que cada uno de } \\
\text { ellos y ellas vive es una de las limitantes. }\end{array}$ & \\
\hline Otros factores & & $\begin{array}{l}\text { A pesar de la iniciativa de las docentes, se } \\
\text { evidencia dificultad para desarrollar los } \\
\text { planes de una forma menos tradicional. }\end{array}$ \\
\hline
\end{tabular}

Nota. Propiedad de Hidalgo K., Navarro V y Rodríguez R. (2012)

La información recolectada muestra que el estudiantado requiere mayor tiempo al realizar las actividades. Además se evidencia que, en su mayoría, no recuerda la información vista en clases anteriores, por lo que el tema debe retomarse en el aula y, en algunos casos, vuelve a ser como un tema nuevo. Se limita, así, el avance de algunos estudiantes que sí recuerdan la información. Esta situación podría mejorarse si se divide el grupo estudiantil en equipos de trabajo que avancen sobre un mismo tema a diferentes velocidades.

Por otra parte, la forma en que se estructura el programa y las situaciones individuales de los estudiantes y las estudiantes hacen que no siempre asistan a clases cada día, por lo que los avances se producen lentamente.

Una de las situaciones que produce preocupación es que aunque se evidencia iniciativa por parte de las docentes para realizar actividades más concretas y lúdicas, se observa dificultad en el desarrollo de planeamientos que respondan más a la diversidad del estudiantado, así como dificultad en la adaptación de materiales tanto para las actividades, como de acuerdo con los niveles de funcionamiento de los estudiantes y las estudiantes. 
URL: http://www.una.ac.cr/educare

CORREO: educare@una.cr

Posteriormente, las docentes y las investigadoras realizaron sugerencias para el desarrollo de procesos similares al realizado en la presente investigación. A continuación se denotan las sugerencias más relevantes.

Tabla 3

Recomendaciones que se identifican de la reflexión y análisis del proceso de mediación pedagógica según docentes e investigadoras

\begin{tabular}{|c|c|c|}
\hline Categorías & Docentes & Investigadoras \\
\hline $\begin{array}{l}\text { Ritmos y estilos de } \\
\text { aprendizaje }\end{array}$ & $\begin{array}{l}\text { Tomar en cuenta el tiempo estipulado } \\
\text { para las actividades ya que los } \\
\text { estudiantes y las estudiantes requieren } \\
\text { tiempo para realizar las tareas. } \\
\text { Elaborar un plan en el que se incluyan } \\
\text { tanto las áreas de matemáticas como de } \\
\text { español, que abarque en los contenidos } \\
\text { ambas áreas, ya que hay muy poco } \\
\text { tiempo para ver tantabmateria. } \\
\text { Mayor información sobre técnicas y } \\
\text { material en concreto para desarrollo de } \\
\text { las clases. }\end{array}$ & $\begin{array}{l}\text { Se requiere más atención individual } \\
\text { para los estudiantes y las estudiantes. } \\
\text { Conocer más del desempeño de los } \\
\text { estudiantes y las estudiantes y sus } \\
\text { habilidades. }\end{array}$ \\
\hline Nivel cognitivo & $\begin{array}{l}\text { Reforzar más el área de lectoescritura ya } \\
\text { que es la de mayor debilidad para ellos } \\
\text { y ellas. }\end{array}$ & $\begin{array}{l}\text { Utilizar técnicas de estimulación } \\
\text { como cuentos y adivinanzas u otras } \\
\text { actividades que ayuden a ejercitar la } \\
\text { memoria. } \\
\text { Organización y planificación para } \\
\text { ejecutar los contenidos }\end{array}$ \\
\hline Intereses y motivación & & $\begin{array}{l}\text { Realizar actividades introductorias para } \\
\text { motivación de los estudiantes y las } \\
\text { estudiantes. }\end{array}$ \\
\hline
\end{tabular}

Nota. Propiedad de Hidalgo K, Navarro V y Rodríguez R. (2011)

Para mejorar la propuesta se debe disponer de un banco de recursos didácticos y materiales concretos de fácil acceso en el aula. Por otra parte, es importante elaborar un plan global con inclusión de actividades que tomen en cuenta los contenidos de las áreas de español y matemáticas, pues el tiempo disponible no es suficiente para concluir los contenidos individualmente.

Por otro lado, se requieren más actividades, en el aula, que estimulen procesos cognitivos favorecedores de las destrezas necesarias para el desarrollo de la lectoescritura.

Finalmente, es necesaria una atención más individualizada para el estudiantado, en la cual se adapten los contenidos y materiales que se utilizarán en las actividades. Se 
debe elaborar, para cada estudiante, un control de avance individual, en donde se anote el progreso de acuerdo con los objetivos planteados, las habilidades desarrolladas y adquiridas.

\section{Conclusiones y recomendaciones}

Se evidencia que la enfermedad mental repercute en el proceso de aprendizaje de los estudiantes del programa de Educación Abierta ofrecido en el Hospital Psiquiátrico en el nivel de alfabetización, en especial en los procesos cognitivos como atención, concentración, memoria a corto y largo plazo. Estas necesidades educativas deben ser consideradas en el quehacer educativo, por lo que es necesario adaptar los contenidos y objetivos curriculares con el fin de facilitar y precisar las áreas académicas en las que se requiere dar mayor apoyo.

Así mismo, el programa de Educación Abierta no ha planificado o establecido procedimientos que aseguren la efectividad de la educación que se ofrece en este lugar, como la revisión de planeamientos a las docentes o la confección de un expediente acumulativo del rendimiento académico de los estudiantes. Si bien es cierto, originalmente, el programa de Educación Abierta estaba pensado para personas que no pudieron terminar sus estudios, es necesario reestructurar el programa para que responda a las necesidades reales de la población que atiende, no solamente para maximizar la inversión económica que se ha hecho, sino también para promover en los estudiantes conocimientos que puedan ser aplicados a su realidad.

Por otra parte, considerando las necesidades que tiene el programa, se determina fundamental la participación de docentes en el área de educación especial, con el fin de dar aportes a las docentes que laboran con los estudiantes, en especial en la utilización de estrategias de intervención pedagógicas adaptadas a las necesidades educativas y a la diversidad de aprendizajes de esta población. Por ejemplo, se evidenció en el transcurso del estudio que a las docentes de I y II ciclos se les dificultó ofrecer una atención individualizada, por lo cual se trabaja los temas con la misma metodología para todo el grupo.

El programa de Educación Abierta es una opción educativa para los usuarios y usuarias de los albergues del Consejo Nacional de Rehabilitación y Educación Especial, así como para las personas que viven en el Hospital Nacional Psiquiátrico. Esta oferta puede proveerles, a estas personas, herramientas muy valiosas para su reinserción social y laboral. Sin embargo, es necesario repensar la situación actual del programa y proveer cambios significativos. Las mediciones pedagógicas realizadas en conjunto con las entrevistas y las observaciones permiten inferir que la labor que se realiza en el programa es muy necesaria e importante, pero existen fallas sistémicas que no siempre benefician a la población que recibe este servicio. 
URL: http://www.una.ac.cr/educare

CORREO: educare@una.cr

\section{Referencias}

Arroyo, L. y Chávez, M. (2001). Discriminación en el derecho a la educación de los niños y niñas. (Tesis de licenciatura inédita), Universidad de Costa Rica, San José, Costa Rica.

Asamblea Legislativa de Costa Rica (29 de mayo de 1996). Ley 7600 Igualdad de oportunidades para las personas con discapacidad. Diario Oficial La Gaceta 112, pp. 1-17.

Asamblea Legislativa de Costa Rica (29 de setiembre de 2008). Ley 8661: Aprobación de la convención sobre los derechos de las personas con discapacidad. Diario Oficial La Gaceta 187, pp. 1-65.

Cabada, J. M. (3 de noviembre de 2004). La atención educativa a la diversidad. Comunidad escolar. Periódico digital de información educativa. 12(753). Recuperado de http:// comunidad-escolar.cnice.mec.es/753/tribuna.html

Elliot, J. (2005). La investigación-acción en educación. (5ª ed.). Madrid, España: Editorial Morata.

Giné, C. (s. f.). Inclusión y sistema educativo. España: Universidad de Salamanca. Recuperado de www.usal.es/ inico/actividades/actasuruguay2001/1.pdf

Lamas, H. y Murrugarra, A. (s. f.). Educación inclusiva. Perú: Sociedad Peruana de Resiliencia. Recuperado de http://www.pasoapaso.com.ve/GEMAS/gemas_264.htm

Ministerio de Educación Pública. (1994). Resumen de política educativa hacia el siglo XXI. San José, Costa Rica: Autor.

Molina, S. (2003). Educación especial. Bases metateóricas e investigadoras. Granada, España: Editorial Ariel.

Organización de las Naciones Unidas para la Educación la Cencia y la Cultura (UNESCO). (2000). Marco de Acción de Dakar. Educación para todos: Cumplir nuestros compromisos comunes. Revista Latinoamericana de Estudios Educativos, 30(2), 133-140. Recuperado de http:// redalyc.uaemex.mx/redalyc/src/inicio/ArtPdfRed.jsp?iCve=27030206\&iCveNum=964

Organización Mundial de la Salud (OMS). (2006). Manual de recursos de la OMS sobre salud mental, derechos humanos y legislación. Ginebra, Suiza: Ediciones OMS.

Organización Panamericana de la Salud (OPS) y Organización Mundial de la Salud (OMS). (2009). Resolución del Consejo Directivo CD 49.R.17. Washington, DC. Recuperado de http:// new.paho.org/hq/dmdocuments/2009/CD49.R17\%20\%28Esp.\%29.pdf

Organización Panamericana de la Salud (OMS). (2010). Resolución del Consejo Directivo CD 50.R.8. Washington, DC. En línea en http://www.un.org/spanish/disabilities/documents/ 
URL: http://www.una.ac.cr/educare CORREO: educare@una.cr

\section{CD50.R8-s.pdf}

Pinto, J. (s. f). Educación especial. Necesidades educativas especiales. Madrid, España: Editorial Imserso.

Sánchez, A. (1997). Intervención psicopedagógica en educación especial. Barcelona: Editorial Universitat de Barcelona.

Sandoval-Chacón, C. y Canales-Víquez, G. (2009). Funcionamiento psicosocial de personas con enfermedad mental desinstitucionalizadas: Una experiencias de rehabilitación psicosocial costarricense. Revista Costarricense de Psicología, 29(41-42), 121-135.

Williamson, G. (setiembre, 2007). Diversidad en la educación: fundamentos, reflexiones y propuestas. Sembrando Ideas Revista Educativa, 1, 57-65. Recuperado de http://issuu.com/ villarricauc/docs/2007/1\#share

\section{Cómo citar este artículo en APA:}

Hidalgo, K., Navarro, V. y Rodríguez, M. R. (2013). La mediación pedagógica como una estrategia para la atención educativa de las personas con enfermedad mental en el Programa de Educación Abierta, en el nivel de alfabetización que se imparte en el Hospital Nacional Psiquiátrico. Revista Electrónica Educare, 17(1), 141-155. Recuperado de http://www.revistas.una.ac.cr/index.php/EDUCARE/issue/current

Nota: Para citar este artículo en otros sistemas puede consultar el hipervínculo "Como citar el artículo" en la barra derecha de nuestro sitio web:

http://www.revistas.una.ac.cr/index.php/EDUCARE/index 